Voix et Images

voixetimages

\title{
L’écrivain est un cénobite, entrevue avec Jacques Ferron
}

\section{Jacques Pelletier et Pierre L’Hérault}

Volume 8, numéro 3, printemps 1983

Jacques Ferron

URI : https://id.erudit.org/iderudit/200396ar

DOI : https://doi.org/10.7202/200396ar

Aller au sommaire du numéro

\section{Éditeur(s)}

Université du Québec à Montréal

\section{ISSN}

0318-9201 (imprimé)

1705-933X (numérique)

Découvrir la revue

\section{Citer ce document}

Pelletier, J. \& L'Hérault, P. (1983). L'écrivain est un cénobite, entrevue avec Jacques Ferron. Voix et Images, 8(3), 397-405. https://doi.org/10.7202/200396ar d'utilisation que vous pouvez consulter en ligne.

https://apropos.erudit.org/fr/usagers/politique-dutilisation/ 


\title{
L'écrivain est un cénobite entrevue avec Jacques Ferron
}

\author{
par Jacques Pelletier, Université du Québec a Montréal \\ et Pierre L'Hérault, Université Concordia
}

\section{L'ÉCRITURE ET LA FOLIE}

V.I. Depuis la publication de Du fond de mon arrière-cuisine en 1973, vous avez observé un certain silence. Puis, vous avez publié l'an dernier Rosaire. Dans le texte qui précède Rosaire, I'Exécution de Maski, référence est faite à une double crise, sur le plan personnel et sur le plan littéraire. Est-ce que ces deux crises sont liées?

J.F. Eh bien non. Ce que j'ai raconté dans l'Exécution de Maski, c'est quelque chose de neuf qui faisait partie d'un roman que j'ai raté. Rosaire est simplement un journal que j'avais tenu en traitant un patient au début des années 60 . II développe à peu près le même thème que Cotnoir mais basé sur des faits et écrit avec beaucoup moins de facilité. Parce que tes personnages dans la réalité ne t'obéissent pas aussi bien que dans les romans. II n'y a pas beaucoup d'invention dans ce roman. Je voulais me remettre à l'écriture. C'est fait. Actuellement, je refais le livre raté, te Pas de Gamelin.

V.I. Dans /'Exécution de Maski, est-il exact de voir une sorte de réflexion sur ce silence de sept ans?

J.F. C'est l'histoire d'un suicide qui n’a pas réussi. Après ça, mon personnage est devenu trop présent. Je ne pouvais parler que de moi. Ça me semblait odieux.

V.I. Est-ce que Rosaire ne représente pas quelque chose de nouveau, justement dans la mesure où il met en scène un personnage différent?

J.F. Non, c'est un journal que j'ai tenu. Rosaire s'appelait Martin Chénard, c'était vraiment un plâtrier qui ne faisait plus de plâtre. Sa femme voulait le placer à SaintJean-de-Dieu pour avoir une pension de nécessiteuse. Ça se situe en 1960, c'est daté. J'ai fait quelques retouches tout simplement.

V.I. Il y a un travail d'écriture; ce n'est pas simplement la transcription d'un journal?

J.F. C'est surtout une transcription de journal.

V.I. Vous rapprochiez tantôt Cotnoir et Rosaire; est-ce que vous voulez dire que la même matière a servi pour Cotnoir? 
J.F. Je ne me suis rendu compte que par après, que je reprenais Cotnoir.

V.I. Est-ce qu'en écrivant Rosaire, vous n'avez pas voulu faire la preuve d'un certain échec de l'imaginaire?

J.F. C'est plutôt l'Exécution de Maski qui représentait pour moi un échec. J'étais pris avec moi-même. Je me détestais. Rosaire est un texte bien antérieur et très simple. Il présente une nouvelle manière de décrire les choses basées sur mon expérience et sans...

V.I Sans contrôle sur les personnages, c'est ça?

J.F. Oui, quoique dans le Pas de Gamelin, qui relate mon expérience à Saint-Jeande-Dieu, je suis nécessairement obligé de généraliser, parce que c'est immense. Les fous, par définition, ne sont pas des gens "normaux". Ils exigent qu'on s'occupe d'eux individuellement. C'est évidemment très fatigant et ils le savent. Ca limite nécessairement ce que tu peux en dire, car ils ne peuvent pas communiquer.

V.I. A partir de 1970, la folie est un thème central de votre oeuvre. Est-ce qu'on peut lire dans la récurrence de ce thème une réorientation majeure de votre écriture qui signalerait une diminution de la préoccupation pour la politique et, une importance plus grande accordée à l'individuel?

J.F. Effectivement. J'ai été plongé dans l'univers de la folie grâce au docteur Trempe. $J$ 'ai travaillé au Mont-Providence, ensuite à Saint-Jean-de-Dieu. Un an et demi à chaque place.

Au Mont-Providence, je me suis fait la main. Je ne connaissais pas tellement la maladie mentale. C'est de cette expérience que j'ai tiré l'Amélanchier, roman sur le drame intellectuel que peut représenter l'enfance et sur l'amnésie qui peut occasionner une perte d'identité si elle n'est pas levée par ce que j'appelle une "mémoire extérieure", c'est-à-dire par un alentour qui soit constant, qui rappelle l'enfant à lui-même chaque jour.

A Saint-Jean-de-Dieu, j'étais plus compétent et j'ai exercé une certaine autorité. $J$ 'étais en charge de la salle d'oligophrénie. Évidemment, ce n'était pas celle où l'on traitait la belle et flamboyante folie; c'était celle où l'on soignait la débilité mentale. Il y avait des folles récaicitrantes qui ne voulaient pas guérir, dont on se débarrassait en les envoyant à l'unité $\mathrm{C}$. J'ai exercé un véritable pouvoir ayant la responsabilité de la salle des femmes - 350 patientes - . Le docteur Trempe, chef de toute l'oligophrénie, me laissait parfaitement libre.

Quand j'ai voulu écrire un livre, j’ai été extrêmement embarrassé; il faut se mettre dans la folie pour en parler, autrement on parle d'une façon arrogante, soit pour culpabiliser les autres, soit pour s'impatroniser, pour montrer quel type prodigreux on est. Se posait aussi la question de l'évasion. Quand un lecteur voit un personnage enfermé, il n'a de cesse qu'il ne soit libéré. C'est l'un des grands moteurs de la littérature que les histoires d'évasion, comme en témoigne la Chartreuse de Parme. Donc il ne fallait pas tomber dans tous ces travers-là. Mais quo farre' J'ai donc écrit dans un embarras complet le Pas de Gamelin. Ca été le premier livre que j'ai raté. 
C'était un livre de fou. Les livres précédents étaient différents. Je faisais un livre, je le remettais à l'éditeur. On le prenait tel qu'il était. Je n'ai jamais eu de conseils de qui que ce soit. Ca se comprend un peu parce que j'ai commencé à publier à compte d'auteur et ensuite j'avais une manière telle que les éditeurs ne me demandaient jamais de reprendre quoi que ce soit. Pourtant plusieurs manuscrits auraient pu ètre corrigés. Enfin...

V.I. Vous avez écrit quelque part: "Ce qui est essentiel, c'est le rapport de soi aux autres". Cela n'indique-t-il pas aussi une préséance de l'individuel sur le collectif?

J.F. Bien sûr! Il est arrivé que dans le feu de la politique, quand j'étais à Parti pris, je me suis mis à penser que c'est le rapport de soi aux autres qui est essentiel; qu'on ne peut pas du tout être sûr que les autres, qu'on voit de l'extérieur, sont semblables à nous, qui sommes à l'intérieur de notre imaginaire. Pour circuler en société, il faut se prendre pour un autre, imaginer qu'on se voit de l'extérieur.

Je me souviens qu' au Mont-Providence j'avais rencontré un psychiatre français. Je lui avais dit: "Pour être normal, il faut se prendre pour un autre". "Mais non, mais non" avait-il protesté. Mais pourtant, il faut absolument, pour être un homme comme tous les autres, s'apercevoir de l'extérieur, être vu et regardé, ne pas être enfermé à l'intérieur de cette coquille où nous sommes uniques, absolument uniques. Sinon, on risque de se replier sur soi, d'entreténir un goût excessif pour la morbidité.

V.I. Vous avez dit tantôt que les gens normaux se cataloguent mais les psychiatres, eux, ont tendance à vouloir faire la même chose avec les fous.

J.F. C'est juste. On ne veut pas affronter le fou, qui est un homme intact, qui a sa biographie propre; on veut le classer dans une catégorie pour pouvoir soigner un schizophrène, un maniaco-dépressif, etc. II me semble pourtant que chaque individu déclaré fou reste un homme intègre, qu'il faut communiquer avec lui, savoir qui il est, d'où il vient, connaître sa biographie, etc. C'est là qu'il faut trouver l'instant où il a déraillé.

V.I. Est-ce que la folie ne pourrait pas être le symbole de la situation que vit le Québécois à l'intérieur des structures qui sont les siennes?

J.F. Oui, et à partir de cette hypothèse on pourrait peut-être expliquer le phénomène de la tuberculose! C'est une maladie que contractent les singes en cage et non en liberté. Dans ma famille, du côté de ma mère, j'ai pu observer des cas: une cousine germaine de ma mère, ses deux soeurs, ma mère elle-même sont mortes de la tuberculose. II y a eu une exception: la cousine Annette qui a fui, s'en est allée à Toronto. Là, comme ça n'allait pas très bien, elle a voulu se suicider. Elle s'est jetée devant une voiture. L'Anglais qui conduisait a freiné, s'est précipité au-devant d'elle, l'a prise dans ses bras et l'a épousée. Elle a été heureuse. Aujourd'hui c'est une dame très correcte...

V.I. Dans les Roses sauvages il y a une phrase qui dit que l'écriture est une sorte de thérapie. Est-ce qu'il s'agit d'un aphorisme qui s'appliquerait à certains cas particuliers d'écrivains, ou l'écriture est-elle toujours pour vous d'une certaine façon reliée à la folie? 
J.F. Oui, l'écrivain est évidemment un cénobite. Il est dans la solitude lorsqu'il écrit; il ne vit absolument pas; on ne garde aucun souvenir des heures passées à écrire, on écrit dans le vide, il faut donc avoir une espece de motivation un peu folle.

\section{L'ÉCAITURE, LE POUVOIR ET LE NATIONALISME}

V.I. Dès que René Lévesque a été porté au pouvoir, vous avez semblé vous désintéresser de la question politique. Comment expliquez-vous cette volte-face?

J.F. C'est simple. Il est arrivé un événement qui $m$ 'a détourné du politique, du moins pour un temps. J'ai tenté de me suicider le vendredi 13 août 1976. Je me suis raté, mais comme le suicide est une chose grave, il faut que tu passes en psychia trie. J'ai alors réalisé un peu le rêve qui me hantait depuis queique temps en me situant dans la folie au lieu de la voir de l'extérieur comme un bel esprit. A la suite de ma tentative ratée, je me suis fait traiter par un psychiatre anglophone. A ce moment-là, je ne voulais voir personne, je ne pouvais rien dire à personne, les preuves d'affection m'affligeaient parce que je ne pouvais les rendre; le psychiatre me faisait signer des petits contrats, des contrats de vie de trois mois; je me suis laissé prendre au jeu et pendant ce temps mes enfants achevaient de se libérer.

V.I. Dans l'appendice aux Confitures aux coings, vous avez écrit: "Le passé, je l'évoque un peu trop, en quoi je me juge réactionnaire". Par contre, vos lecteurs ne vous ont pas pris pour un réactionnaire, mais plutôt pour un écrivain bien ancré dans la problématique actuelle du Québec. Comment conciliez-vous ces deux perceptions de votre oeuvre?

J.F. Au fond je n'ai jamais pensé être réactionnaire. Dans le passé, nous avons toujours été en lutte avec les gens qui disaient: "le national ça ne compte pas, ce qui est important c'est le social, réglons le problème du chômage..."; du chômage il y en a toujours eu et il ne se serait absolument rien fait si on ne s'était occupé que du social; alors, oui, réactionnaire aux yeux de tous ces gens-là qui veulent tout faire passer par le social.

V.I. N'y a-t-il pas cependant dans votre oeuvre une certaine nostalgie de la société traditionnelle?

J.F. II y a un anthropologue américain qui s'appelle Sapir, un ami de Marius Barbeau, qui a écrit en 1926: "Bien sûr les besoins matériels et les nécessités de la vie seront fournis par des organismes internationaux, tout nous porte vers ça, mais la seule façon d'empêcher l'entropie, la dissolution du pareil au même, c'est de susciter les différences culturelles dans le monde." Moi, je suis devenu nationaliste simplement pour une question de langue. Je suis allé en Gaspésie j'ai fait mes études à Québec - et j’ai remarqué, auprès de mes malades, que les gens du bas de Québec parlaient bien et puis en haut de Québec pas si bien. Evidemment ce fut une révélation: des gens qui parlaient français d'une façon naturelle tout en étant souvent illettrés, n'ayant pas à chercher leurs mots, parlant une belle langue verte! Ensuite, venant à Ville Jacques-Cartier, j'ai vu autre chose et $j$ 'a réalisé que deux langues comme le français et l'anglais ne peuvent pas coexister. Et le danger est que la langue verte, source de notre français, se corrompe et disparaisse, comme c'est arrivé à Lowell. 
V.I. Ȧ partir de ce que vous venez de dire, est-ce qu'on ne pourrait pas affirmer que l'écriture qui conteste le pouvoir est du côté de la culture orale?

J.F. Oui, sincèrement, je crois à ça. Vous savez, l'écrivain ne fait pas dès livres parce qu'il a lu d'autres livres - bien sûr, ça donne des idées... - mais, s'il veut faire un livre qui soit du pays, il faut qu'il parte de la tradition orale. Et si tu as une langue verte qui se corrompt comme à Lowell, il est préférable - comme Jack Kerouac l'a fait dans le Docteur Sax - d'écrire en anglais. S'il n'y a pas une tradition orale, la langue est morte.

V.l. Vous êtes donc devenu nationaliste pour des raisons qui n'ont rien à voir avec l'économie?

J.F. Remarquez que les raisons économiques peuvent entrer en ligne de compte. C'est la nationalisation de l'électricité qui a permis à René Lévesque, du temps des libéraux, de faire parler français à l'électricité. Ça m'a frappé beaucoup.

V.I. Á propos de la crise d'octobre 1970, vous avez été le premier, du moins le premier personnage connu, à soutenir une thèse, développée ensuite par Vallières, qui prétend que les événements ont été manipulés par la police.

J.F. Peut-être pas simplement par la police mais par les sociologues américains qui avaient voulu étudier la question du Chili. Le Chili avait refusé. Enfin je crois qu'à partir de 1963 tout a été manipulé. Je connaissais par exemple Mario Bachand. Il était prisonnier et, en finissant son terme, il a pu s'envoler vers Saint-Pierre-etMiquelon, ce qui n'était pas du tout dans ses moyens et pas dans ceux de la petite organisation à laquelle il appartenait. II y avait quelqu'un pour l'aider. A Saint-Pierre-et-Miquelon, il pouvait mettre la France dans l'embarras; on s'est aperçu immédiatement qu'il y avait en dessous de ça une instance qui ne nous était pas propice et, de fait, ca s'est terminé par une espèce de grande crise de terrorisme.

V.I. Est-ce qu'on ne peut pas en tirer la conclusion que les revendications des Québécois ne peuvent aller au-delà d'un certain seuil sans provoquer une réaction de répression de la part non seulement du gouvernement fédéral mais aussi du gouvernement américain?

J.F. Chose certaine, les choses se sont faites d'une façon très lente, très correcte avec un minimum de pertes de vie. On avait sans doute imaginé des scénarios. Puis il y a eu la mort de Laporte qui m'engueulait a l'Assomption parce que je n'étais pas nationaliste. On lui a dédié des écoles, en même temps qu'on faisait circuler des rumeurs sur ses relations présumées avec la pègre. Mais le terrorisme s'est arrêté là. Quelques soubresauts avec l'affaire du malchanceux agent Samson. Puis le silence... La violence ne fait pas partie de nos habitudes. Nous sommes une nation pacifique qui n'a pas connu la guerre, qui est née sous la domination étrangère, qui a progressé et qui n'a jamais été portée à la violence.

V.I. II reste que dans les années 1960, dans le climat d'effervescence politique d'alors, ce type d'engagement apparaissait logique.

J.F. On ne peut nier en tout cas que Paul Rose, excellent maitre d'école en enfance 
inadaptée, soit passé à la dissidence en 1968 après une manifestation. C'est à ce moment que sont apparus les premiers prisonniers politiques parce que $n$ 'importe quel individu, sur les lieux, qui ne pouvait pas dire: "J'allais porter ceci à ma grand-mère" était condamné à la prison. II y en a eu plusieurs qui se sont fait arrêter et jeter en prison. Une institutrice de nos amis n'a pu nier être sur les lieux, elle ne pouvait donc pas avoir d'autres raisons que de participer à ce qui était devenu une émeute. C'est à ce moment-là que Rose s'est écoeuré et qu'il a commencé sa grande lutte avec Jacques Lanctôt, lequel était malheureusement connu comme terroriste depuis 1963 et dont le père, Paul, était bon ami avec le ministre de la justice d'alors, Rémi Paul... Voilá pourquoi l'auteur des Deux solitudes a pu dire: "C'etait d'authentiques nazis". II $y$ avait un petit fondement là à cause de Rémi Paul et de Lanctôt qui est le petit-fils de Charlie Lanctôt (Charlie Lanctôt qui a fait tomber Taschereau) et le fils de Paul Lanctôt qui était un lieutenant d'Adrien Arcand.

\section{DE LA LITTÉRATURE QUÉBÉCOISE}

V.I. Comment voyez-vous la littérature québécoise actuelle?

J.F. Je la vois de moins en moins puisque je lis de moins en moins. Elle est d'une nouvelle génération que je connais peu. J'ai lu quand même d'assez beaux livres et, en général, je crois que le niveau intellectuel est beaucoup plus élevé. On le voit par les lettres aux journaux; autrefois on était peu nombreux à écrire, il $n^{\prime} y$ avait pas de concurrence, à présent c'est occupé par une grande confrérie. Quant à la littérature, elle m'apparaît d'une grande richesse. On voit apparaitre des best-sellers - comme chez les Américains qui produisent des choses sérieuses, faites pour le marché, faites pour être vendues - on voit apparaître ça chez nous، c'est un phénomène nouveau.

\section{V.I. Beauchemin?}

J.F. Beauchemin... Effectivement, it a pris une stature internationale qu'on n'avait pas. Godbout, par exemple, publie au Seuil mais ses livres se vendent surtout au Canada... Et en même temps, il $y$ a ceux qui font des recherches formelles et qui publient des choses dont je dois convenir qu'elles sont intéressantes.

V.I. Par rapport à ce qui se fait maintenant, avez-vous conscience d'avoir joué un rôle dans le déblocage de la littérature québécoise?

J.F. On me.le laisse croire! Je me suis quand même toujours situé pàrmi les écrivains mineurs. Les écrivains mineurs peuvent être utiles mais ils disparaissent, bien qu'ils puissent nourrir une littérature majeure.

V.I. A vous entendre et à vous lire, on a l'impression que vous considérez que votre influence s'est fait sentir surtout sur le terrain de l'histoire nationale dont vous avez renouvelé la conception en même temps que vous remettiez le projet national à l'ordre du jour.

J.F. Oui, en ressortant les drapeaux... J'ai fait mon travail d'historien sérieusement: j'en surs satısfait bien que ce ne soit pas un travail qui appartienne à la grande littè ature. Enfin j'étais pris par un Québec que j'aurais mieux aimé si la langue 
n'avait pas été menacée. J'aurais parlé de choses ordinaires comme on le fait dans les autres pays.

V.I. Vous faites remonter I'histoire du Québec au début du XIXe siècle, c'est-à-dire au moment où on prend conscience que nous formons une nation différente. Est-ce que la naissance de notre littérature se situe à la méme époque?

J.F. Les grands écrivains du XIXe siècle ont été des historiens comme Garneau ("T'as menti, Durham") et après eux, les auteurs de récits de voyage, partant à la conquête du monde et rapportant une partie du monde à leurs lecteurs. Évidemment il y a eu de la poésie, des romans à cette époque-là, mais ça ne pesait pas lourd.

V.I, II y avait aussi reprise des querelles littéraires françaises, des luttes d'école dans le contexte québécois.

J.F. Ah! bien sûr... Nous étions pris dans une littérature absurde, dans une pensée d'exportation. Nous étions, par exemple, grands défenseurs du Pape qui était contre le principe des nationalités alors que c'était notre principe vital. De fait, le seul type dont on a gardé mémoire parmi tous ces zouaves-là, c'est aussi le seul qui soit allé se battre pour la liberté: Arthur Buies.

V.I. Pour en revenir à vous, ne pourrait-on pas dire que les plus jeunes ont trouvé dans votre oeuvre la preuve qu'on pouvait produire une oeuvre littéraire à partir de notre histoire et notre patrimoine?

J.F. Oui, lorsque je suis sorti de Brébeuf, je me souviens très bien qu'écrire, dans un texte, "rue Sainte-Catherine", ça me semblait une impossibilité, ça ne faisait pas partie du langage littéraire. Mes premiers textes sont empreints de cette conception; je pense par exemple à la Barbe de François Hertel: ça se passe à Paris. Or, je n'ai jamais voulu aller à Paris. Je suis allé en Pologne, j’ai fait un arrêt à Orly, j'ai vu une petite rivière, c'est tout. Non, la France est un pays qui né peut pas nous être propice. Nous devons beaucoup aux Français, ils nous ont apporté des travaux fondamentaux, comme par exemple la géographie de Blanchard, I'Homme et I'Hiver de Desfontaines. Ce sont des livres que les Francais sont venus faire ici, ils nous ont donné des instruments de travail. L'ancienne littérature du Régime Français a eu beaucoup d'influence sur la France, sur l'Enclopédie - c'est Claude Roy qui a dit que la Bastille était tombée sous le coup de la ruée des Hurons, des Iroquois, des Canadiens... - en apportant un modèle de civilisation differente de celle qu'on croyait.

V.I. Est-ce qu'il y a des écrivains français ou québécois qui ont été importants pour vous?

J.F. Bien sûr. Au collège, avant la guerre, en 39, j'étais abonné à la Nouvelle Revue française; c'était là que se publiait la grande littérature. Je ne savais pas que c'était mon maître, mais il y avait un homme que j'observais beaucoup à l'époque, Pierre Baillargeon qui, lui, a décidé de faire carrière littéraire alors que pour moi ce choix était impossible. Mon père n'aurait pas compris. Et puis il fallait que je gagne ma vie. Mais lui, il s'est lancé dans la littérature et, finalement, 
il n'a pas eu la liberté d'écrire ce qu'il voulait et il est mort assez tristement. Toupin et Baillargeon avaient pour modèle Berthelot Brunet, un notaire qui me fascinait. Ils ont eu de l'importance pour moi parce qu'ils avaient décidé de vivre des lettres, mème en quémandant, alors que moi je ne l'avais pas fait.

V.I. Valéry, auteur qui fascine votre personnage dans la Charrette, il vous a intéressé aussi?

J.F. Oui. Nous sommes passés par une littérature tout à fait desséchante, mal arrosée, celle de Valéry; mon personnage de la Charrette parle de Valéry mais comme quelqu'un d'une autre génération, comme à l'époque de Duplessis on aurait pu parler de Horace ou de Virgile.

\section{L'OEUVRE}

V.I. Quand on analyse le Ciel de Québec, on se rend compte qu'il y a six ou sept intrigues qui évoluent parallèlement avec des croisements ici ou là. Aviez-vous des plans pour mener toutes ces intrigues de front?

J.F. C'est assez bizarre. Au moment de la rédaction du Ciel de Québec, je sortais de Mont-Providence ou j'avais travaillé beaucoup. Je me suis retrouvé dans mon petit cabinet. J'avais des loisirs. Jacques Hébert m'avait dit: "à Francfort, nous avons l'air fou, nous ne présentons que des galettes". Alors j'ai voulu faire un gros livre.

V.I. II me semble que le livre que vous aimez le plus dans votre oeuvre soit la Charrette; pourquoi?

J.F. C'est un livre que j'ai écrit à la mort de ma soeur Thérèse que j'aimais beaucoup. Enfants nous étions très attachés. Ce livre-là, oui, c'est un livre que j'aime beaucoup mais qui est peut-être un peu difficile.

V.I. Votre préférence pour la Charrette tient-elle à sa forme ou à son contènu?

J.F. J'ai voulu faire un peu le bilan de mes pensées sur le parti communiste. II y a là un personnage - Marsan - à qui Bèlial veut acheter le restant de son âme... et ça se passe dans une géographie que je connais bien. C'est un livre vite fait, j'ai toujours aimé les livres vite faits. Claude Hurtubise de la maison $\mathrm{HMH}$ m'avait demandé un inédit pour la réédition de mes contes. J'ai donc écrit rapidement la Charrette pour satisfaire à sa commande.

V.I. Vous avez écrit il y a quelques années: "je crois qu'il faut gagner son sel et qu'il n'est pas question de faire le métier d'écrivain; écrire ce n'est pas une profession, la preuve c'est qu'il n'y a pas de diplôme d'écrivain et, s'il y en avait, ce serait la fin de la littérature". Au moment où il y a des écrivains québécois qui commencent à vivre de leurs livres, est-ce que vous voyez les choses toujours de cette façon-là?

J.F. J'ai insisté sur le droit d'écrire qui me semblait similaire au droit de parole. J'ai toujours eu beaucoup d'admiration pour les écrivains qui ont réussi à vivre de leur plume. J'ai signé récemment le certificat de décès de Jovette Bernier qui a réussi à faire fortune et à vivre de ses livres. J'admire aussi Thériault parce 
qu'il s'est donné du mal, qu'il a beaucoup travaillé. D'ailleurs l'argent que je touche comme médecin, ça ne me fait pas grand plaisir, c'est de l'argent que j'aime gagner, mais l'argent que je touche comme écrivain, j'en suis fier.

V.I. Autrement dit, votre position était d'une certaine façon dictée par la nécessité?

J.F. Oui, oui, c'est ça. II n'était pas question pour moi de devenir un écrivain profes-: sionnel, d'abord parce qu'au début, je ne savais pas quoi dire. Avant d'aller en Gaspésie, je pouvais faire des phrases brillantes mais ça n'avait pas de portée.

V.I. Vous parlez de vos premières pièces, par exemple?

J.F. Oui, des trucs comme ça et un roman que je n'ai pas publié parce que je le trouvais insignifiant.

V.I. Quand vous dites que vous préférez l'argent qui vient de la vente de vos livres plutôt que de votre profession de médecin, est-ce que vous voulez dire que vous placez l'écriture plus haut que la médecine dans votre échelle de valeur?

J.F. Vous savez, en médecine, je suis un peu athée...

V.I. Pour finir, on peut dire que vous voyez votre oeuvre comme détachée, en morceaux, d'une certaine façon?

J.F. J'ai toujours fait mes livres en toute liberté, sans trop de difficultés. Je devais d'ailleurs écrire très vite car je n'avais guère le temps. Évidemment un grand écrivain ne peut pas écrire comme ça. Quand il entre dans son oeuvre, il ne doit pas en sortir: il faut du temps, c'est comme ça. Juste des incursions de quelques semaines, ce n'est pas suffisant pour qu'une oeuvre mûrisse. 